R. Sadanandan, M. Stöhr, W. Meier, Simultaneous OH-PLIF and PIV measurements in a gas turbine model combustor, Appl. Phys. B 90 (2008) 609618.

The original publication is available at www.springerlink.com

http://dx.doi.org/10.1007/s00340-007-2928-8 


\title{
Simultaneous OH-PLIF and PIV measurements in a gas turbine model combustor
}

\author{
R. Sadanandan ${ }^{\star}$, M. Stöhr, W.Meier \\ Institut für Verbrennungstechnik, DLR Stuttgart, Pfaffenwaldring 38-40, 70569 Stutttgart, Germany.
}

Received: date / Revised version: date

\begin{abstract}
Simultaneous PIV and OH-PLIF measurements were conducted in an enclosed gas turbine model combustor for investigating the influence of turbulence on local flame characteristics. The non-premixed swirling $\mathrm{CH}_{4}$ /air flame that was investigated had a thermal power of $10.3 \mathrm{~kW}$, an overall equivalence ratio of $\phi=0.75$ and exhibited thermoacoustic oscillations at a frequency of approximately $295 \mathrm{~Hz}$. The flame was lifted and burnt partially premixed. Different superequilibrium $\mathrm{OH}$ concentration levels were observed in the measurements. Calculations using the PREMIX code with GRI 3.0 mechanism revealed that the different $\mathrm{OH}$ levels are a strong function of the local $\phi$ and depend much less on the degree of premixing of $\mathrm{CH}_{4}$, air and hot gas. Reaction zones were identified by large gradients in the single shot OH-PLIF distributions. The measurements revealed the formation of reaction zones at regions of high flow velocities where the hot burned gas from the inner or outer recirculation zones mixes with the fresh fuel/air mixture at the burner exit. Though the reaction zones are continuous over a few centimeters like in flamelet regime, there exist regions where the mixture has failed to ignite, possibly due to the high local strain rates present, emphasizing the complex turbulence-chemistry interactions leading to finite rate chemistry effects. The instantaneous PIV images showed the existence of small vortical structures close to the shear layers much in contrast to the time averaged PIV images where the flowfields were typical of enclosed swirl burners, namely an inner recirculation zone and an outer recirculation zone. These small vortical structures were seen to play a vital role in the formation and destruction of reaction zone structures. The reaction zones were mostly formed at the interface between the hot and cold gases with the relative orientation of the reaction zone being parallel to the flowfield. However, in some regions the reaction zones were also seen to orient orthogonally to the flowfield without being disturbed by the high velocity flow.
\end{abstract}

PACS $33.20 ; 33.50 ; 47.27 ; 47.32 ; 47.70 ; 82.33 ; 82.40$

\footnotetext{
^ Fax: +49-711-6862 578, E-mail: rajesh.sadanandan@dlr.de
}

\section{Introduction}

Combustion under gas turbine (GT)-like conditions is a very complex process governed by a complicated turbulent flowfield and strong effects of turbulence-chemistry interactions. Modern stationary gas turbines are operated in a lean premixed or partially premixed mode. However, premixing is not perfect because usually fuel and air are mixed shortly before entering the combustion chamber leading to a significant degree of unmixedness [1,2]. Flame stabilization is most often achieved by swirl which induces a recirculation of burned gas to the flame root thereby mixing hot gas and radicals with fresh gas. Thus, a variety of different mixtures of fuel, air and exhaust gas can be present before flame reactions start [1,3]. Mixtures with high temperatures can spontaneously ignite if the ignition delay time is on the order or shorter than the typical time scale of the flowfield. After ignition a flame front can propagate through a partially premixed gas containing fuel and oxygen until the flame front is extinguished, e.g. by high local strain rates. The situation becomes even more complex if thermoacoustic instabilities occur which further influence mixing and reaction [4-8]. Considering this complex interplay between flowfield, chemical reactions and (acoustic) boundary conditions, it becomes clear that the theoretical understanding of such flames is still incomplete and that its numerical simulation is challenging. In order to improve the understanding, experiments with advanced laser techniques are needed.

A main advantage of laser techniques is their ability to yield two-dimensional fields of velocities and scalars with high temporal and spatial resolution. For the 2D measurement of the flowfield, particle image velocimetry (PIV) or stereoscopic PIV are well suited and for the imaging of scalars planar laser induced fluorescence (PLIF) is mostly used [9]. In particular, the simultaneous application of PIV and PLIF enables new insights into the interaction between a turbulent flowfield and chemical reactions. Of special interest is here the visualization of the reaction zones by, e.g. PLIF of OH or $\mathrm{CH}$. Several research groups have contributed to a better understanding of various aspects of turbulence-flame front in- 
teractions by applying the combination of these techniques. Frank et al. used PLIF of OH together with PIV to study counter-gradient transport in reaction zones of premixed turbulent $\mathrm{CH}_{4}$ /air flames [11]. Similar investigations have been performed by Böhm et al. in a partially premixed counterflow flame [12]. The flame-vortex interaction was studied by Meyer et al. applying OH PLIF/PIV in a counterflow flame [13]. Driscoll and coworkers applied PIV simultaneously with PLIF of $\mathrm{OH}$ or $\mathrm{CH}$ to characterize flame extinction phenomena in turbulent non-premixed jet flames [14,15]. Rehm and Clemens used PIV/OH-PLIF [16] and Han and Mungal [17] used PIV/CH-PLIF to study the influence of strain on the reaction zones in non-premixed jet flames. Watson et al. investigated the flame stabilization of a lifted $\mathrm{CH}_{4}$ /air diffusion flame by CH-PLIF/PIV [18]. Hult et al. used the multi-shot imaging setup in Lund together with stereoscopic PIV to study the interaction between the reaction zone and turbulent vortices in the DLR jet flame [19]. A flame with a more complex flowfield was investigated by Petersson et al. [20]. They applied PIV, PLIF and filtered Rayleigh to the premixed unconfined low swirl flame from the Lawrence Berkeley National Laboratory. Tanahashi et al. used $\mathrm{CH}$ and OH-PLIF simultaneously with stereo PIV to study the flame structures in a confined swirl-stabilized premixed flame [21].

In the experiments presented here, simultaneous $\mathrm{OH}-$ PLIF/PIV imaging was applied to a confined partially premixed GT model flame. The GT model combustor has been used before for the investigation of different $\mathrm{CH}_{4}$ /air swirl flames [3, 22-30]. The flame used in this investigation has a thermal power of $10 \mathrm{~kW}$ and a global equivalence ratio of $\phi=0.75$ and exhibits thermoacoustic pulsations at a frequency of around $290 \mathrm{~Hz}$. In previous studies this flame has been characterized using laser Raman scattering, laser Doppler velocimetry, PLIF of $\mathrm{OH}, \mathrm{CH}$, and $\mathrm{H}_{2} \mathrm{CO}$ and twoline OH-PLIF thermometry. Thus, a huge amount of experimental data is available to support the interpretation of the current experimental results.

In this paper, we concentrate on the flame structures and on the interaction between the flowfield and the reaction zones. The different levels of $\mathrm{OH}$ concentrations occurring in this flame and the identification of the reaction zones by the gradients of the $\mathrm{OH}$ distributions are discussed. Furthermore, the different orientations of the reaction zone with respect to the flowfield are treated. The main goals of this contribution are the demonstration of the potential of simultaneous PLIF/PIV measurements for the investigation of GT-relevant flames and to contribute to a better understanding of the combustion process in these flames.

\section{Experimental Details}

\subsection{Gas Turbine Model Combustor and Flames}

The schematic of the Gas Turbine (GT) model combustor and the burner used for the measurements is shown in Fig. 1. The burner is a modified version of a GT combustor originally equipped with an air blast nozzle for liquid fuels [31].
Co-swirling dry air at room temperature and atmospheric pressure was supplied to the flame through a central nozzle (diameter $15 \mathrm{~mm}$ ) and an annular nozzle (i.d. $17 \mathrm{~mm}$, o.d. $25 \mathrm{~mm}$ contoured to an outer diameter of $40 \mathrm{~mm}$ ). Both air flows were fed from a common plenum with an inner diameter of $79 \mathrm{~mm}$ and a height of $65 \mathrm{~mm}$. The radial swirlers consisted of 8 channels for the central nozzle and 12 channels for the annular nozzle. The ratio of the air mass flows through the annular and central nozzle was approximately 1.5 . Nonswirling $\mathrm{CH}_{4}$ was fed through 72 channels $(0.5 \times 0.5 \mathrm{~mm})$, forming a ring between the air nozzles. Compared with an annular nozzle for $\mathrm{CH}_{4}$ with a slit width of $<0.5 \mathrm{~mm}$, this configuration ensured better realisation of the cylindrical symmetry of the fuel injection and sets well-defined boundary conditions for numerical simulation. The exit planes of the fuel and central air nozzles were located $4.5 \mathrm{~mm}$ below the exit plane of the outer air nozzle; the latter was defined as reference height $h=0$. A contoured steel top plate with a central exhaust tube (diameter $40 \mathrm{~mm}$, length $50 \mathrm{~mm}$ ) formed the exhaust gas exit. The combustion chamber had a square section of $85 \times 85 \mathrm{~mm}$ and a height of $114 \mathrm{~mm}$ and was equipped with 4 quartz windows held by 4 rods (diameter $10 \mathrm{~mm}$ ) in the corners, thereby enabling excellent optical access to the flame zone. The high velocity in the exhaust tube avoided any backflow from outside the combustion chamber.

The $\mathrm{CH}_{4}$ /air flame that was investigated in the current studies had an overall equivalence ratio $\phi=0.75$ and thermal power $P_{t h}=10.3 \mathrm{~kW}$, resulting from a $\mathrm{CH}_{4}$ mass flow of $12.3 \mathrm{~g} / \mathrm{min}$ and air mass flow of $280.8 \mathrm{~g} / \mathrm{min}$. The $\mathrm{CH}_{4}$ and air flow rates were regulated with high precision coriolis (Siemens Mass 2100) and mass flow controllers (Bronkhorst). The flame under these operating conditions was lifted and thus partially premixed before ignition and it exhibited thermoacoustic oscillations at a frequency of approximately $295 \mathrm{~Hz}$. This frequency did not correspond to any resonance frequency of the combustor geometry. The pressure variations were measured by two microphones (Brüel \& Kjaer 4939), located at the plenum and in the combustion chamber at a height of $16 \mathrm{~mm}$ in one of the 4 rods that held the quartz windows. The nozzle Reynolds number based on the cold inflow and the minimum outer nozzle diameter $(25 \mathrm{~mm}$ ) was about 15,000 and the swirl number of the burner calculated from the velocity profile just above the burner was $S \approx 0.55$ [30]. From the visible appearance and photos from different directions the flame exhibited axial symmetry despite of the square combustion chamber. However, from PIV measurements it could be deduced that the flowfield exhibited a slight deviation from axial symmetry close to the combustor walls induced by the corners of the chamber.

\subsection{Experimental Set-up and Measurement Techniques}

The schematic of the optical set-up used for the measurements is shown in Fig. 2. It is a combination of an OH-PLIF system with a PIV system. The output beams from the dye 


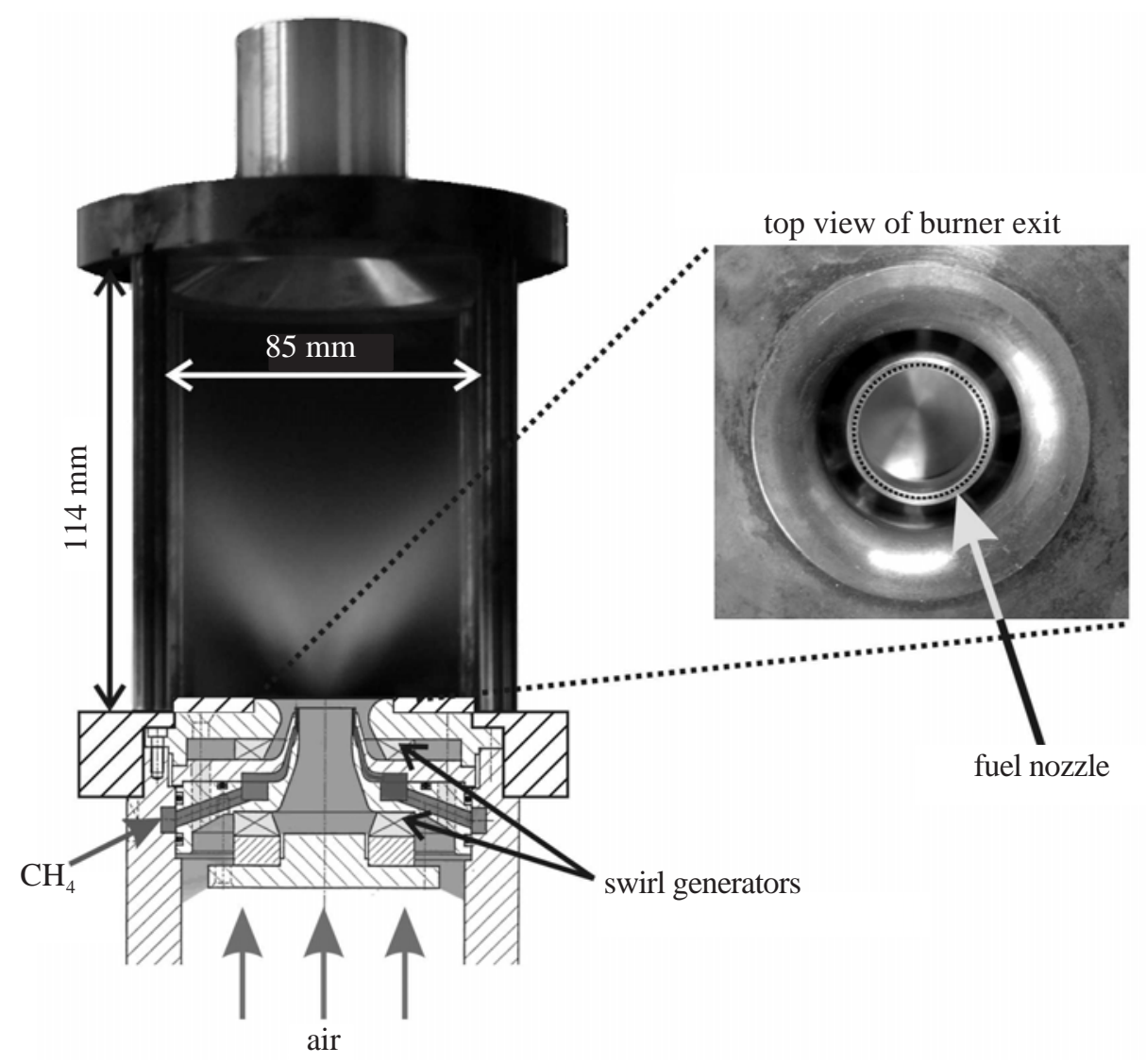

Fig. 1 Schematic drawing of the GT model combustor together with a photograph of the combustion chamber; Inset: zoomed view of the burner as seen from the top.

laser at $283 \mathrm{~nm}$ for the OH-PLIF excitation and the double pulse Nd:YAG laser at $532 \mathrm{~nm}$ used for the PIV measurements were expanded into a vertical sheet by means of a series of cylindrical lenses as shown in Fig. 2. Both sheets were then combined together and were then sent along the same optical path into the combustion chamber. This was achieved by means of a mirror with high reflectivity at $283 \mathrm{~nm}$ and also high transmissivity at $532 \mathrm{~nm}$. The OH-PLIF and PIV cameras were positioned diametrically opposite to each other with respect to the combustion chamber. So no image dewarping was performed as the image distortion due to perspective projections and inherent camera lens distortions were very minimal. The pixel to pixel spatial matching between the two cameras was accomplished by imaging a common reference grid placed at the same plane as the laser sheets at the burner exit. From these images the pixel/mm ratio for each camera was calculated to recalibrate all LIF and PIV images and to map the two camera images into a common coordinate system.

2.2.1 OH-LIF System: For the OH-PLIF measurements a flashlamp pumped, frequency doubled Nd:YAG laser (Quanta Ray, DCR-2) was used to pump a frequency doubled tuneable dye laser (Lumonics, HD-500) at $10 \mathrm{~Hz}$. The UV beam (pulse duration $8 \mathrm{~ns}$ and line width $\approx 0.4 \mathrm{~cm}^{-1}$ ) had a pulse energy of approximately $2.5 \mathrm{~mJ}$ at the exit of the laser and was tuned to the $\mathrm{Q}_{1}(8)$ transition of $\mathrm{OH}$ at approximately $283 \mathrm{~nm}$ in the $v^{\prime \prime}=0, v^{\prime}=1$ vibrational band of the $A^{2} \Sigma^{+}-X^{2} \Pi$ system. The population of the laser-coupled ground state of the selected line varied by approximately $9 \%$ in the temperature range of interest $(1400 \mathrm{~K}-2200 \mathrm{~K})$. Subsequent $\mathrm{OH}$ fluorescence in the $v^{\prime}=1, v^{\prime \prime}=1$ and $v^{\prime}=0, v^{\prime \prime}=0$ bands near $310 \mathrm{~nm}$ was detected through an interference filter in the wavelength region $295-340 \mathrm{~nm}$. This also enabled suppression of laser light scattered from both OH-PLIF and PIV systems and background radiations. The laser sheet inside the combustor was approximately $50 \mathrm{~mm}$ in height and $400 \mu \mathrm{m}$ in thickness. The spectral laser intensities in the imaged area was approximately $3.1 \mathrm{MW} / \mathrm{cm}^{2} \mathrm{~cm}^{-1}$ for $\mathrm{OH}$. The applied laser intensities were above the saturation intensities of approximately $1 \mathrm{MW} / \mathrm{cm}^{2} \mathrm{~cm}^{-1}$ [32] for the chosen transitions of $\mathrm{OH}$ and thereby a considerable degree of saturation is to be expected in measurements. By means of a beam splitter, a small portion of the incoming laser beam is directed into a $\mathrm{CH}_{4}$ /air reference flame in a McKenna burner regulated by means of digital mass flow controllers, operating under laminar conditions. The LIF produced by the flame is used for the online monitoring of the excitation line wavelength.

The OH-PLIF detection system consisted of an imageintensified CCD camera (Roper Scientific, $512 \times 512$ pixels) with achromatic UV lens (Halle, $f / 2, f=64 \mathrm{~mm}$ ). Part of incoming laser sheet was deflected into a quartz cell filled 


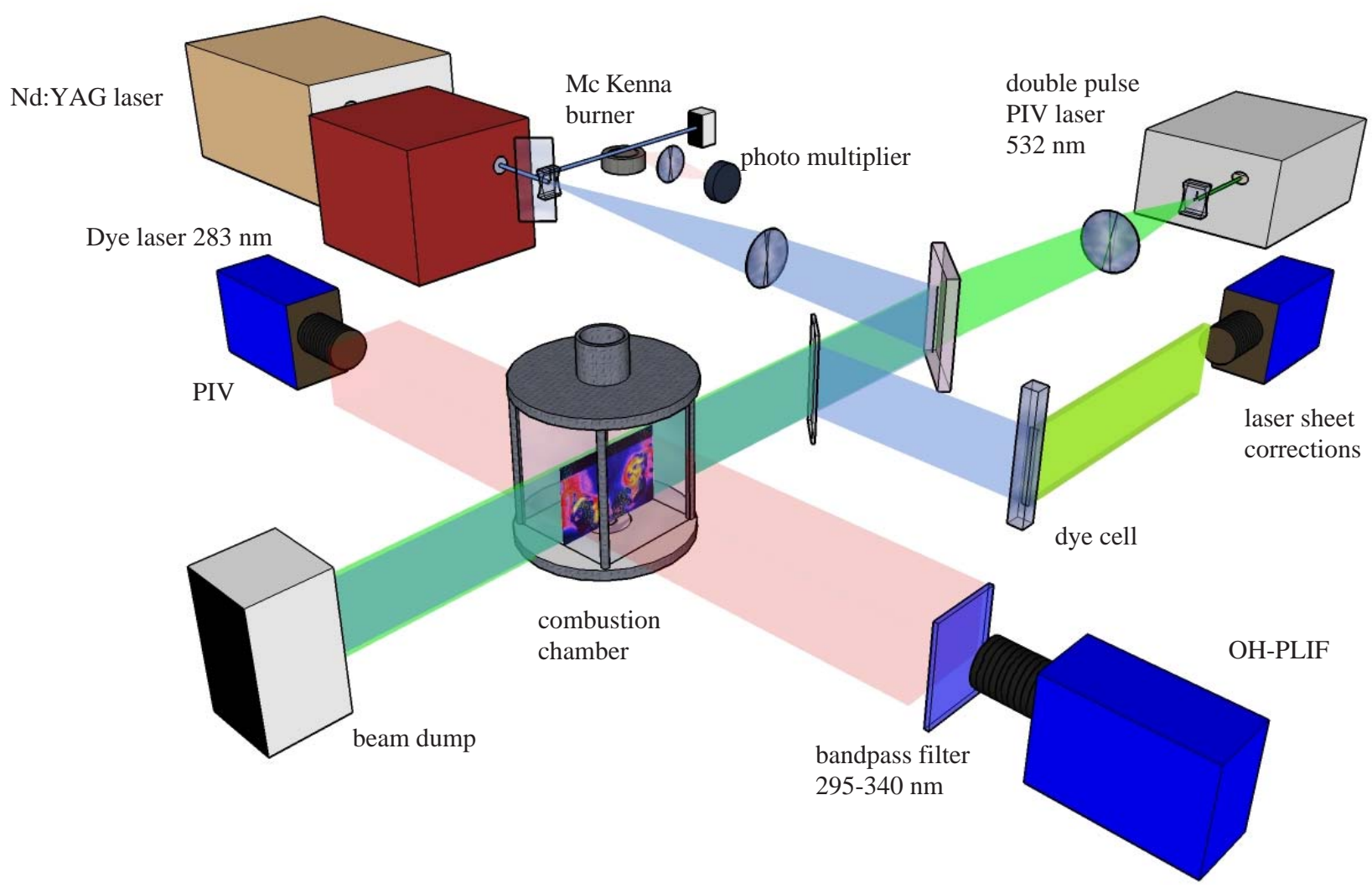

Fig. 2 Schematics of the optical system used for simultaneous OH-PLIF and PIV measurements.

with fluorescent dye solution by means of a beam splitter. The dye cell fluorescence profile was simultaneously imaged using another ICCD camera (Roper Scientific, $512 \times 512$ pixels) equipped with a Nikon lens $(f / 2.8, f=50 \mathrm{~mm})$. This dye cell profile is used for correcting the laser sheet profile inhomogeneities. The image intensifier was set to an exposure time of $400 \mathrm{~ns}$ for both OH-PLIF and dye cell fluorescence cameras. The spatial resolution of the system based on the size of a pixel in the flowfield is approximately $185 \mu \mathrm{m}$. The same detection system along with the filter combination was used for the $\mathrm{OH}^{*}$ chemiluminescence measurements. The exposure time for the image intensifier was set to $40 \mu$ s to capture the integrated (along the line of sight) spontaneous emission from $\mathrm{OH}$ in the electronically excited state $\left(\mathrm{OH}^{*}\right)$.

\subsubsection{PIV System: The PIV system (New Wave Solo PIV} 120) consisted of a frequency doubled double-pulse Nd:YAG laser, a double-shutter CCD camera (LaVision, 1376×1024 pixels) and a programmable timing unit (PTU). The laser had a pulse energy of $120 \mathrm{~mJ}$ per pulse at $532 \mathrm{~nm}$. The vertical sheet had a height of approximately $60 \mathrm{~mm}$ and a thickness of $1 \mathrm{~mm}$ inside the combustor. The time separation between the two laser pulses was $15 \mu \mathrm{s}$. The air flow was seeded with $\mathrm{TiO}_{2}$ particles with a nominal diameter of $1 \mu \mathrm{m}$. The spatial resolution of velocities is $0.77 \mathrm{~mm}$ (corresponding to an interrogation region of $12 \times 12$ pixels) in both directions. The rate of image acquisition was 5 double frames per second. Due to the coating of the windows with seeding particles during the measurements, acquisition of images was possible only for $100 \mathrm{~s}$ corresponding to 500 image pairs. Thereafter the windows had to be cleaned before any further measurements could be performed.

The vector fields and streamline plots were generated using the commercial PIV software from LaVision (FlowMaster). A multi-scale cross-correlation algorithm was used, with the interrogation window size ranging from $64 \times 64$ pixel to $16 \times 16$ pixel. In the hot gas regions, approximately 6 particles were present in an area of $16 \times 16$ pixel, whereas in the unburned gas, the density was considerably higher (more than 12 particles per interrogation area). Spurious vectors were detected using the deviation from the local median, and replaced either by vectors corresponding to the 2 nd, 3 rd or 4 th highest correlation peak (if they fulfil the median criterion), or else by an interpolated vector.

2.2.3 Time synchronisation: The timing between the different laser pulses and the camera gate openings were synchronised by means of a PTU from LaVision and a pulse delay generator (SRS Inc. type, DG532). This enabled synchronising the OH-PLIF and PIV cameras both framing at $2.5 \mathrm{~Hz}$ with the OH-PLIF and PIV laser systems operating at $10 \mathrm{~Hz}$. The OH-PLIF image was captured in between the first and 
the second PIV images at $10 \mu$ s after the first PIV laser pulse. The time delay helped in reducing the effects of laser light scattering from the PIV laser in addition to providing simultaneous OH-PLIF images with the velocity fields.

\subsection{Image Post Processing - Identification of the Reaction Zones from $\mathrm{OH}$-LIF Images}

Using OH-LIF image to locate the transient reaction zones in highly turbulent flows is not always straightforward as detectable amount of $\mathrm{OH}$ exists in abundance not only in the reaction zone but also in the burned gases where the temperature of the gases is approximately above $1400 \mathrm{~K}$. However, the equilibrium $\mathrm{OH}$ concentration increases exponentially with temperature. So, in the reaction zone where the temperature is maximum, one can expect a sharp temperature gradient and a correspondingly sharp OH-LIF intensity gradient. Additionally, chemical kinetic models as well as previous Raman and LIF investigations in jet flames have shown that $\mathrm{OH}$ exists in superequilibrium concentrations in the reaction zone $[33,34]$. This results from the difference in kinetic rates between the two-body radical production reactions and the three-body radical decay reactions towards equilibrium. For atmospheric-pressure flames, the characteristic timescale for two-body and three-body reactions are approximately $20 \mu \mathrm{s}$ and $3 \mathrm{~ms}$ respectively. The strong temperature gradients together with the existence of superequilibrium $\mathrm{OH}$ concentration in the reaction zone results in strong gradients in the $\mathrm{OH}-$ PLIF images. Thus by deducing the gradients of the OH-PLIF distributions in the axial and radial directions, the spatial location and structure of the transient reactions zones can be identified. There are different digital image processing methods available for gradient calculation. In the present work, we used a Sobel gradient filter which takes the derivative in the one direction and smoothes in the orthogonal direction using a one dimensional version of triangular filter [35]. The spatial averaging operation with the triangular filter is based on a local averaging of the input filter where all the values within the filter support have differing weights. This combined process of differentiation and spatial averaging alleviates the sensitiveness of the method to small luminance fluctuations in the image.

\section{Results and Discussion}

\subsection{Flame shape and flowfield characteristics}

The single shot and time averaged streamline plot of the flowfield are shown in Fig. 3. The time averaged plot shows a flowfield typical of enclosed swirl burners with a conically shaped inflow of fresh gas, an inner recirculation zone (IRZ) and an outer recirculation zone (ORZ). A shear layer of high velocity fluctuations is formed between the IRZ and the high velocity inflowing stream. A second shear layer of high velocity fluctuation is formed between the ORZ and the inflow. The instantaneous streamline plot on the other hand shows a completely different picture. It shows the existence of small scale vortical structures at regions close to the shear layers which did not show up in the time averaged images. These small scale vortical structures in the inner and outer shear layer along with the high turbulence intensity present in the shear layer result in intense mixing between the cold fresh gas and the hot burned gases coming from IRZ and ORZ. It can be seen in the following sections that the intense mixing between the hot and the cold gases in turn plays a vital role in the mixing and ignition of the fresh gas and thereby for the stabilisation of the flame above the burner in regions of high flow velocities.

Fig. 4(a) shows the time averaged $\mathrm{OH}^{*}$ (electronically excited) chemiluminescence image of the flame under the same operating conditions. The chemiluminescence image gives information about the shape and position of the heat release zone. When compared to LIF, which samples the electronic ground state of the target species, chemiluminescence offers qualitative information about the concentration of species produced in the excited electronic state far from equilibrium within the flow. The near UV spontaneous emission from $\mathrm{OH}^{*}$ is visible only in the heat release zone and not in the region of burned gases. Since it is a line of sight technique, with the total emission collected along the line of sight, the spatial resolution is compromised. The chemiluminescence distribution displayed in Fig. 4(a) shows a rather flat flame with the main region of heat release between 5 and $25 \mathrm{~mm}$. It is not quite clear whether the slight asymmetry of the distribution is real or artificially caused by the experimental setup, e.g., by a small misalignment of the optical axis. In case of axisymmetric steady flames, the chemiluminescence images can be reprocessed to yield spatially resolved information. Fig. 4(b) shows the deconvoluted chemiluminescence image obtained from the time averaged image. It can be seen that the heat release zone is limited only up to a height of $\mathrm{h} \simeq 35 \mathrm{~mm}$. Also the flame is not attached to the fuel nozzle exit, instead, it stabilises at a height of approximately $2-5 \mathrm{~mm}$, thereby implying a high level of mixing before ignition and that the flame is of partially premixed nature [30].

Fig. 5(a) and Fig. 5(b) displays the time averaged and instantaneous OH-PLIF images of the flame respectively. The laser sheet travels from left to right in the image. The difference in intensities seen between the left half and right half of the OH-PLIF image, in spite of having an axisymmetrical flame, is due to the absorption by the $\mathrm{OH}$ radicals. The absorption determined by comparing the average fluorescence intensities in a rectangular region $(5 \mathrm{~mm}$ wide and $10 \mathrm{~mm}$ in height) from two sides of the combustor over a distance of $5 \mathrm{~mm}$ from the quartz windows at a height of $30 \mathrm{~mm}$ from the burner exit was $\sim 44 \%$. In order to reduce the influence of absorption on the interpretation of the results we depict only sample results from the left side of the combustion chamber $(\mathrm{r}=-40-0 \mathrm{~mm})$ in the following sections. As mentioned above OH-LIF acts as a marker of the regions with high temperature namely, the reaction zone and hot burned gases. The dark region at the immediate exit of the burner represents the cold inflow with mixture temperature less than $\approx 1400 \mathrm{~K}$. On 


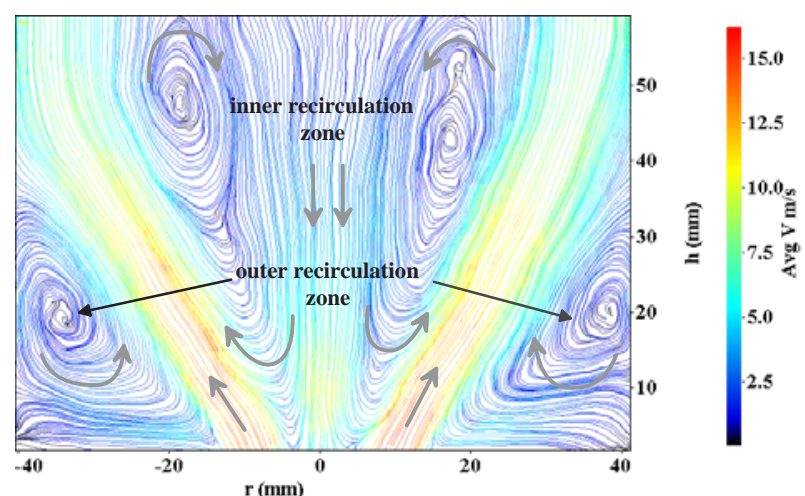

(a)

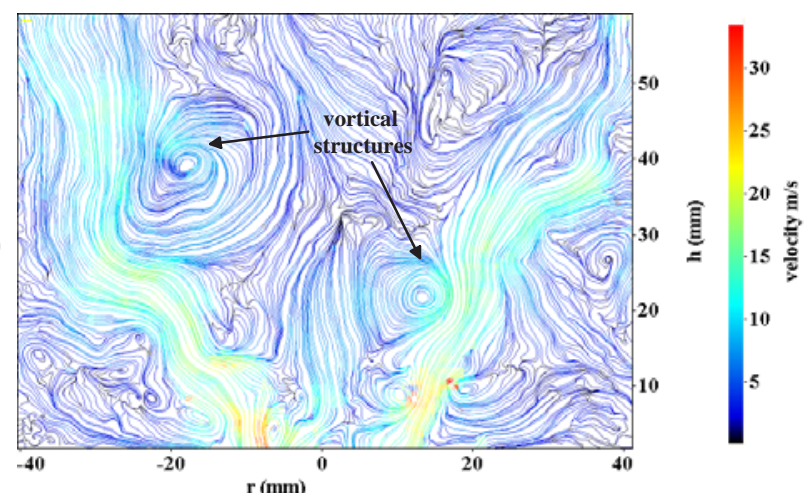

(b)

Fig. 3 (a) Time averaged stream line plot of the flowfield at the axial plane of the burner and (b) Instantaneous stream line plot of the flowfield at the axial plane of the burner.

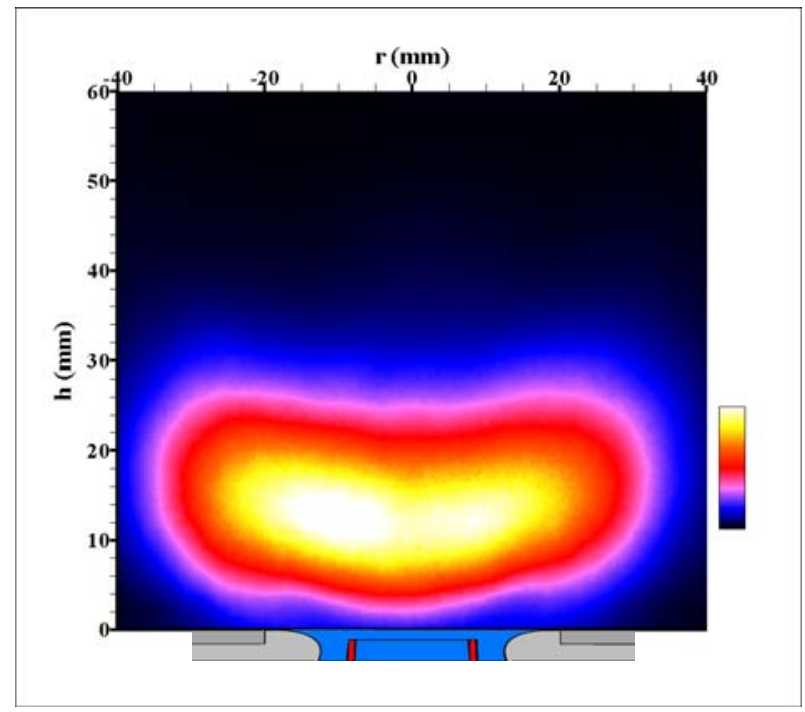

(a)

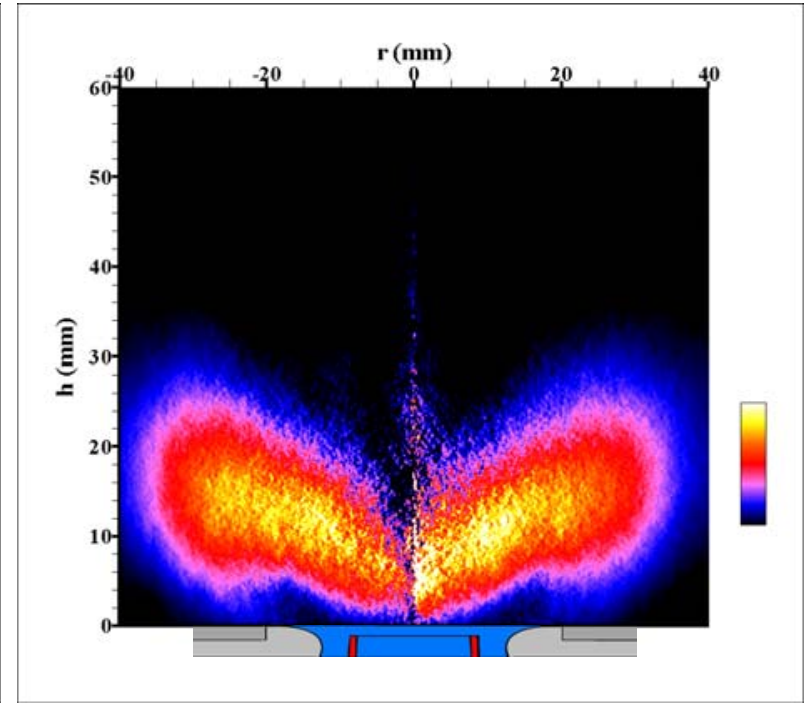

(b)

Fig. 4 (a) Time averaged $\mathrm{OH}^{*}$-chemiluminescence image and (b) deconvoluted $\mathrm{OH}^{*}$-chemiluminescene image.

comparing with the time averaged $\mathrm{OH}^{*}$ image in Fig. 4(a) one can conclude that the LIF intensity above $30 \mathrm{~mm}$ in the instantaneous and time averaged OH-PLIF images are from the hot products of combustion and not from the presence of reaction zone as the heat release zone extends only up to a height of $30 \mathrm{~mm}$.

In the instantaneous OH-LIF image, at the core of the IRZ ( $\mathrm{r}=-10-10 \mathrm{~mm}, \mathrm{~h}>15 \mathrm{~mm}$ ), no strong OH-LIF intensity variations are visible, indicating an absence of reaction zones in this region. Interestingly, the OH-LIF intensity scales are also low in this region. But from previous temperature measurements using planar two-line OH-PLIF thermometry [29] and laser Raman scattering [30] methods in the same combustor geometries and under the same operating conditions, it was seen that the temperature in this region is around $1950 \mathrm{~K}$, with almost negligible rms fluctuations at this height. Considering the rather long distance the fluid elements have passed before they reach the core of the IRZ, it is obvious that the $\mathrm{OH}$ concentrations are at equilibrium in this region. In contrast, in the shear layer between the inflow and the IRZ (around $\mathrm{h}=5-30 \mathrm{~mm}, \mathrm{r}=-25-0 \mathrm{~mm}$ ) high OH-LIF intensities are seen with levels approximately 5 to 7 times higher than in the IRZ. These high levels are indicative of superequilibrium $\mathrm{OH}$, i.e. $\mathrm{OH}$ that has just been formed in a reaction zone or transported only a short distance away from the reaction zone. Further, fuel and air are not mixed to the global equivalence ratio at these positions [3] so that reactions are likely to occur at $\phi \approx 1$ with flame temperature around $2200 \mathrm{~K}$ and correspondingly higher $\mathrm{OH}$ levels than in the IRZ. In order to assess the influence of superequilibrium $\mathrm{OH}$ concentration for different equivalence ratios, calculations for premixed $\mathrm{CH}_{4}$ /air flames have been performed using the "PREMIX" code with the GRI 3.0 mechanism. The calculations have been performed for $\phi=0.75$ and 1.0 for $\mathrm{CH}_{4}$ /air at $\mathrm{T}=300 \mathrm{~K}$. In addition, typical mixtures of $\mathrm{CH}_{4}$, air and exhaust gas in the temperature range from $750 \mathrm{~K}$ to 


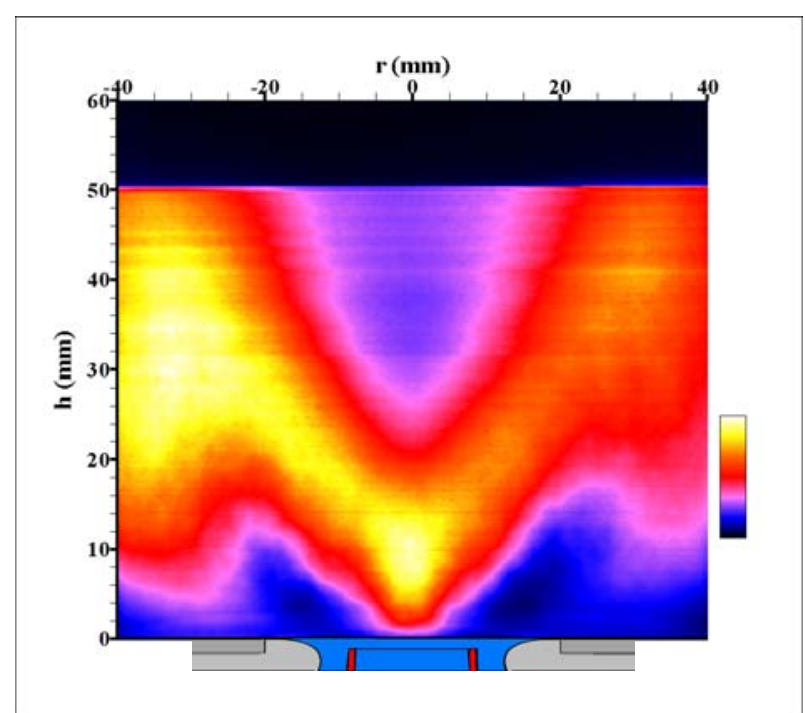

(a)

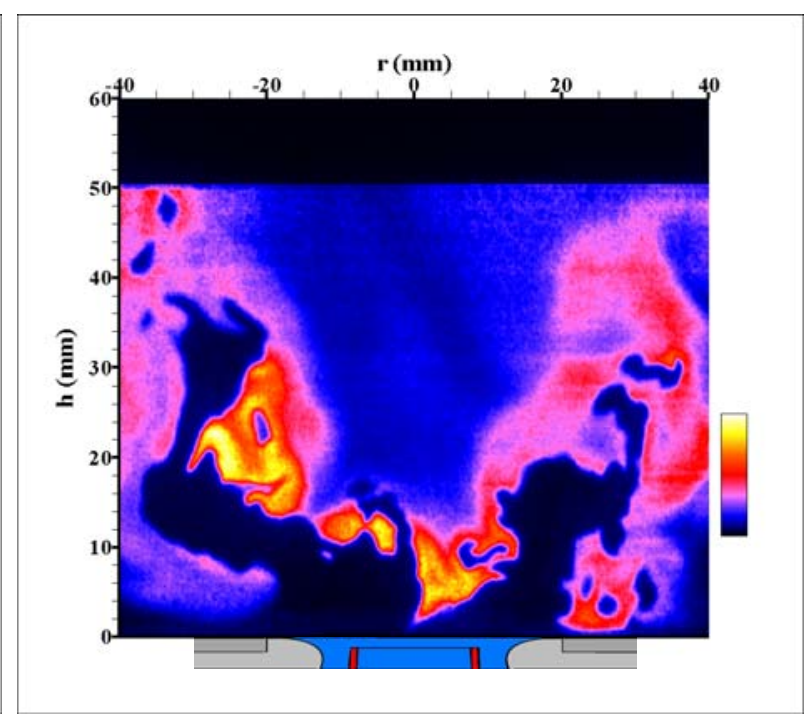

(b)

Fig. 5 (a) Time averaged and (b) instantaneous OH-PLIF distribution of the flame.

$1500 \mathrm{~K}$ were also used as input parameters. These gas mixtures were determined as follows: For the $\phi=0.75$ and 1.0 the adiabatic equilibrium composition, including $\mathrm{NO}$ and the radicals $\mathrm{O}, \mathrm{H}$, and $\mathrm{OH}$, and temperature were calculated. In the next step, several compositions of burnt gas (at adiabatic equilibrium) and fresh gas (at $300 \mathrm{~K}$, same $\phi$ ) were calculated in such a way that the resulting temperatures of the mixtures were $750 \mathrm{~K}, 1000 \mathrm{~K}, 1250 \mathrm{~K}$, and $1500 \mathrm{~K}$. These compositions were taken as the input data for the PREMIX calculation. The resulting OH mole fractions are displayed in Fig. 6 as a function of time. All curves show a fast rise of $\mathrm{OH}$ at the onset of the flame reactions followed by a slower decay towards equilibrium. For $\phi=1.0$ the maximum $\mathrm{OH}$ mole fraction is $\approx 0.0075$ which is about 2.5 times the equilibrium concentration. It is also seen that the different gas mixtures with the same $\phi$ yield nearly the same $\mathrm{OH}$ levels. At $\phi=0.75$ the maximum $\mathrm{OH}$ mole fraction is $\approx 0.005$ and the equilibrium value is $\approx 0.001$. From the Raman measurements it is known that the exemplary gas mixtures chosen for the calculation are representative values in the near field of this flame [3]. Thus, the calculation demonstrates that the $\mathrm{OH}$ level can vary significantly depending on $\phi$ and superequilibrium state. In the core of the IRZ the $\mathrm{OH}$ concentrations are expected to be close to equilibrium and with $\phi \approx \phi_{\text {global }}=0.75$ the $\mathrm{OH}$ mole fraction should be close to 0.001 according to the calculation. In the outer region of the instantaneous OH-PLIF distribution of Fig. 5(b)(r=-30--40 mm) the OH-LIF level is approximately 2-3 times higher than in the IRZ, probably due to the transport of $\mathrm{OH}$ from the reaction zones that has not yet reached equilibrium. For example, for an axial velocity of approximately $15 \mathrm{~m} / \mathrm{s}$ at burner exit, the superequilibrium $\mathrm{OH}$ will travel a distance of approximately $15 \mathrm{~mm}$ from the reaction zone in $1 \mathrm{~ms}$.

Fig.7 shows the corresponding gradients of the OH-LIF distribution in Fig. 5(b). The largest gradients most likely

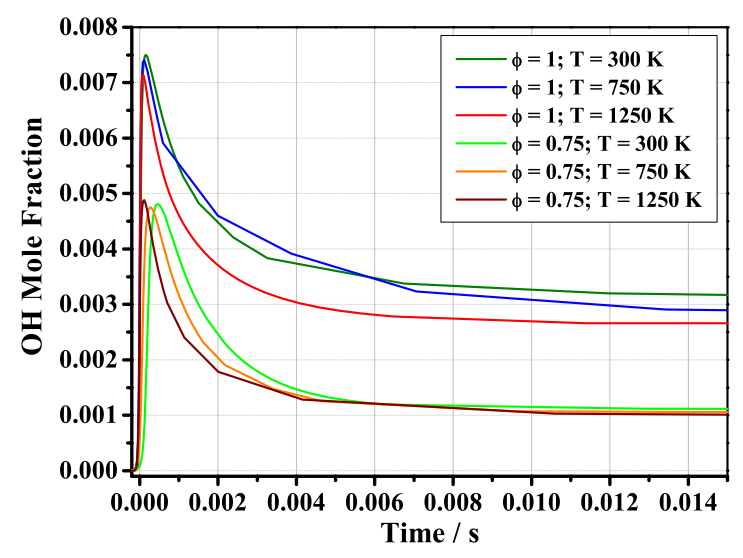

Fig. 6 Dependence of superequilibrium $\mathrm{OH}$ concentration on different temperature and equivalence ratio combinations.

correspond to reaction zones, the smaller gradients to boundary layers between hot and cold gases. This interpretation is, however, subject to some ambiguity because the orientation of the $\mathrm{OH}$ layers with respect to the laser sheet is not known. The angle of intersection between the $\mathrm{OH}$ boundary layer and the laser sheet has, of course, an influence of the deduced gradient. Here, the smaller gradients are masked by selecting a threshold value (5\% of the maximum value, arbitrarily chosen) and displaying only the gradients above this threshold value. From the visual similarity of these images with the CH-PLIF images, commonly used to visualise reaction zones, shown in Fig. 8, measured under identical operating conditions $[24,36]$, it can be seen that it is reasonable to use the gradient OH-PLIF images as an approximate marker of the reaction zone. The strongly wrinkled contours 


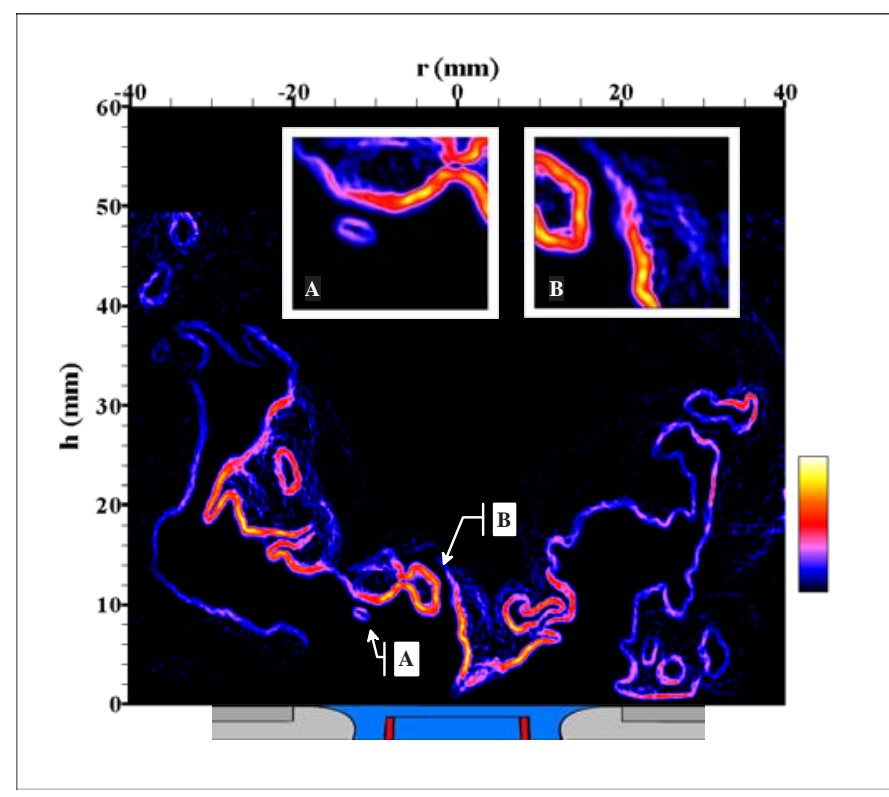

Fig. 7 Approximate spatial position and structure of the reaction zone deduced from the OH-PLIF distribution in Fig. 5(b); Inset: zoomed in views of regions marked $\mathrm{A}$ and $\mathrm{B}$.

in the gradient plot give a good impression of the turbulence present at the burner exit. In most of the single shot OH-PLIF images reaction zones are continuous over a few centimeters in the measured 2D plane like the flames in the flamelet regime. Paths with large gradients (reaction zones) often go over in lines with small gradients where reactions have presumably ceased shortly before the image was taken. Besides regions that are presumably ignition (marked $\mathrm{A}$ ) and extinction (marked B) events were often seen in the single shot OH-PLIF images, highlighting the strong interplay between turbulence and chemical reactions. The "breaks" in the reaction zone can be an outcome of the interaction with small scale vortical structures where the increasing strain can influence the residence times available for thermal runaway and thereby leading to local extinction via finite rate chemistry effects. However, one need to be careful in the interpretation of such images in view of the three dimensional nature of the flow-flame interactions. The diminished gradient seen in the measured 2D plane could also simply mean that the the reaction zone may be connected out of plane. In addition, from the studies by Meier et al. it was seen that the investigated flame (and also other flames typical of gas turbine combustion) exhibit a wide range of mixture fraction and temperature combinations under such operating conditions. These stem from the large variety of mixtures of fuel, air and exhaust gas. As a result of these disparate mixture fraction temperature combinations, reactions can be initiated by spontaneous ignitions and the flame front can move through a premixed gas mixture for some distance, and interact with the flowfield.

\subsection{Simultaneous $\mathrm{OH}-\mathrm{PLIF}$ and PIV Measurements}

One example of a simultaneously measured OH-PLIF and PIV image is shown in Fig. 9(a) with the PIV plot overlapped over the OH-PLIF image. The spatial position and structure of the reaction zone with respect to the flowfield is displayed in Fig. 9(b) where the PIV plot overlapped over the gradient image that was deduced from the same OH-PLIF image. Again, one can see the corrugated shape of the $\mathrm{OH}$ distribution and the different levels of $\mathrm{OH}$ concentrations. Furthermore, one can clearly witness flame stabilisation in regions of high velocities at the burner exit. Reaction zones are formed at the outer and inner shear layers where the hot burned gases from the outer and inner recirculation zones mix with the fresh fuel/air mixture (region $\mathrm{C}$ and $\mathrm{D}$ respectively). However the formation of reaction zones in regions where hot gases mix with fresh fuel/air mixture is not continuous. For example in region $\mathrm{E}$, where almost identical flow conditions exists as in region $\mathrm{D}$, the gradients are weak or the presence of a strong reaction zone is hardly visible. This could be possibly a case of local flame extinction due to the presence of high local strain rates which in turn will result in a relatively small residence time available for a successful kinetic runaway.

Fig. 10 shows another example of simultaneously measured OH-PLIF and velocity fields. Here, a very broad region of high $\mathrm{OH}$ concentration is visible in region marked $\mathrm{F}$. The broad $\mathrm{OH}$ distribution seen here should be due to the transport of "young" $\mathrm{OH}$ at superequilibrium conditions from a nearby reaction zone. This assumption is reasonable as the burner is operated at lean conditions and atmospheric pressure. However, this does not seem to be the case in the region marked $\mathrm{G}$ although one can see a broad region of high $\mathrm{OH}$ concentration in this region also. The direction of the flowfield is from top to bottom and there is no indication of a reaction zone in the upstream of this region when one follows the velocity vectors towards larger heights. But the amount of $\mathrm{OH}$ concentration present in this region, as shown by the colour scale, indicates superequilibrium $\mathrm{OH}$. A reasonable guess is that this $\mathrm{OH}$ stems from a reaction zone outside the LIF plane. However, for a more accurate interpretation of the local flowchemistry interactions, one needs additional information in the third dimension (stereo PIV images for example) or high speed OH-PLIF images.

Fig. 11 show four examples where the influence of flow dynamics on the orientation of the reaction zone is demonstrated. The influence of small scale vortical structures on the structure of the flame front is shown in Fig. 11(a) and (b) where selected streamlines are plotted along with the $\mathrm{OH}-$ PLIF gradient and velocity vectors for better visualisation. The instantaneous streamline plots of the flowfield in Fig. 3(b) showed the existence of small scale vortical structure in regions close to the shear layer much in contrast to the time averaged stream line plots. The small regions of the flame front shown in Fig. 11(a) and (b) show varying levels of interaction of these vortical structures on the reaction zone. For example in Fig. 11(a), the reaction zones lie on the circumference of the vortical structures. Or in other words, there is 


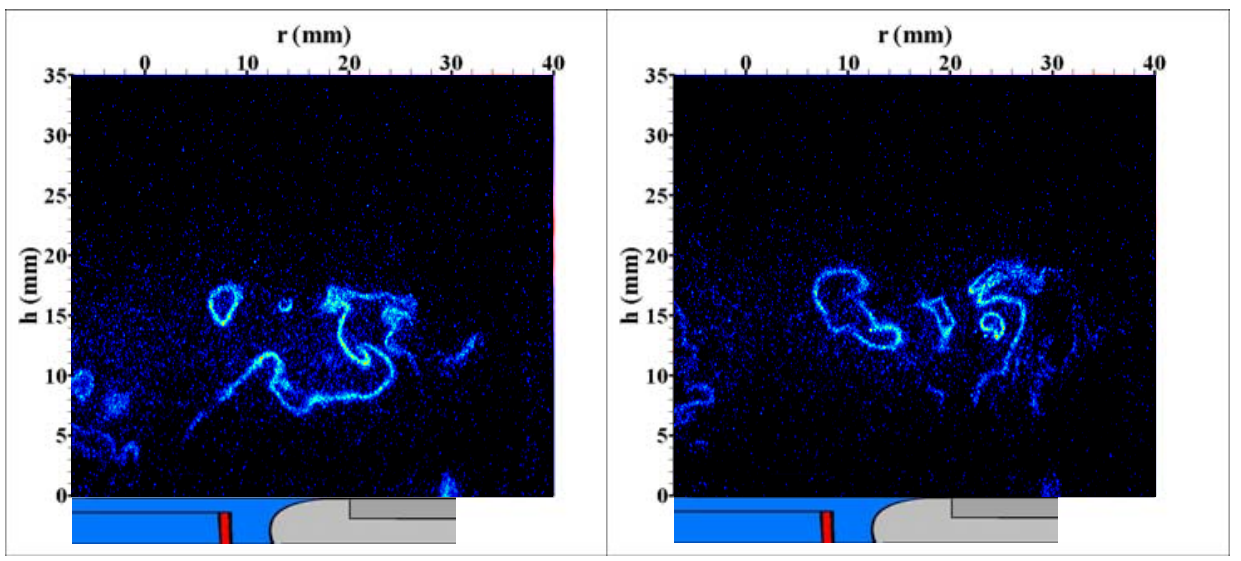

Fig. 8 Two examples of instantaneous CH-PLIF images. For details refer to [24]

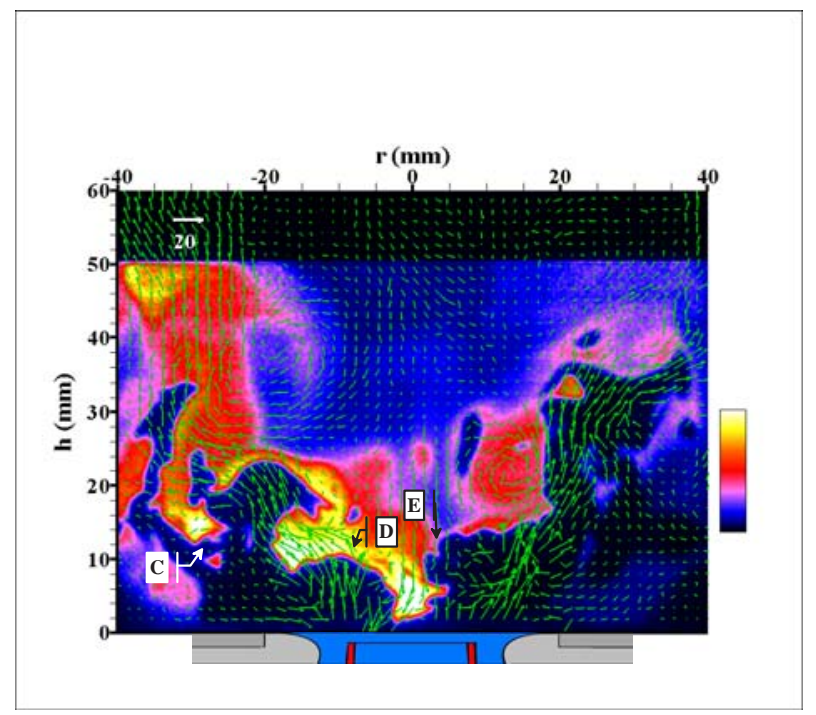

(a)

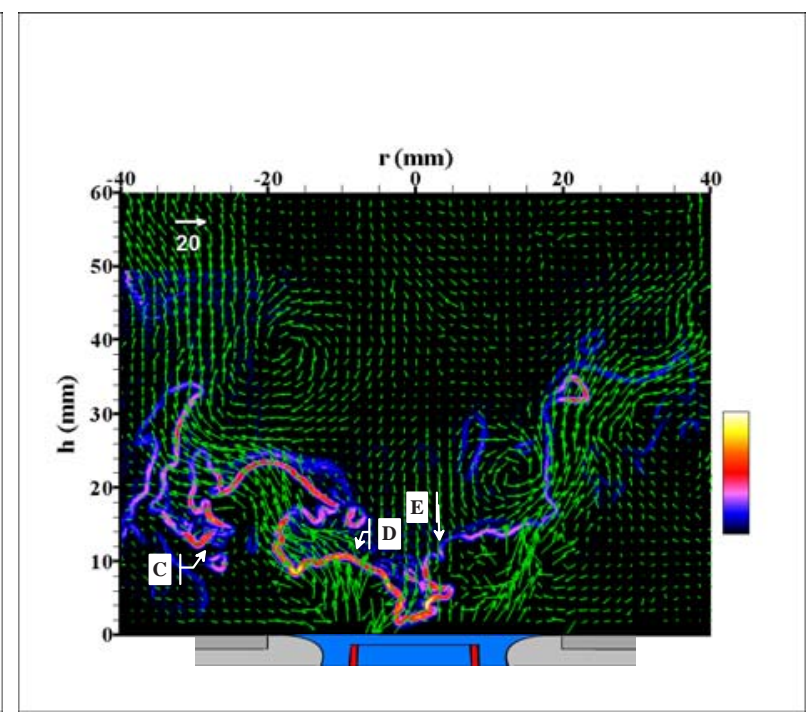

(b)

Fig. 9 (a) Simultaneous OH-PLIF and velocity distributions (b) Velocity distributions overlapped over the gradient image deduced from the same OH-PLIF image.

a roll up of the flame front due to these vortices, there by increasing the flame surface area and correspondingly the reaction rates. Interactions of such nature where a deformation of the flame front with vortical structures occurred were often seen in our studies in the different single shot images. However, Fig. 11(b) shows interactions of different nature. When compared to Fig. 11(a), here the reaction zone appears to have been less affected by the vortex flow. This may be due to the small size of the vortex and the corresponding weak vorticity. For high kinematic viscosities associated with high flame temperature, the local vorticity destruction by local gas expansion could play against the convective roll up process of the flame front by small vortices. The strong vortices can alter the flame front only if they succeed in weakening the flame by stretch effects [37]. Similar findings were reported by Donbar et. al. [15] in turbulent non-premixed jet flames studies where it was seen that small eddies appear to play no role in the wrinkling of the flame surface.
Fig. 11(c) and (d) highlight the different orientations of the reaction zone with respect to the flowfield. In Fig. 11(c) the reaction zone is oriented parallel to the flowfield. Here reaction zone is formed at the interface where the hot gases from the IRZ or ORZ mix with the fresh inflow. This was seen to be the case in most of the instances. However, Fig. 11(d) shows a case where the orientation of the reaction zone is perpendicular to the flowfield direction. At the velocity of $\approx 15 \mathrm{~m} / \mathrm{s}$ prevailing in this region, the reaction zone is convected by the flowfield and flame displacement is of minor importance. Thus, the reaction zone must have been formed shortly before $(\leqslant 1 \mathrm{~m} / \mathrm{s})$ near the burner exit. Because of this short residence time, there must have been high temperatures in the region where the flame front was established. Cases similar to (d) were relatively seldom observed in the measurements. 


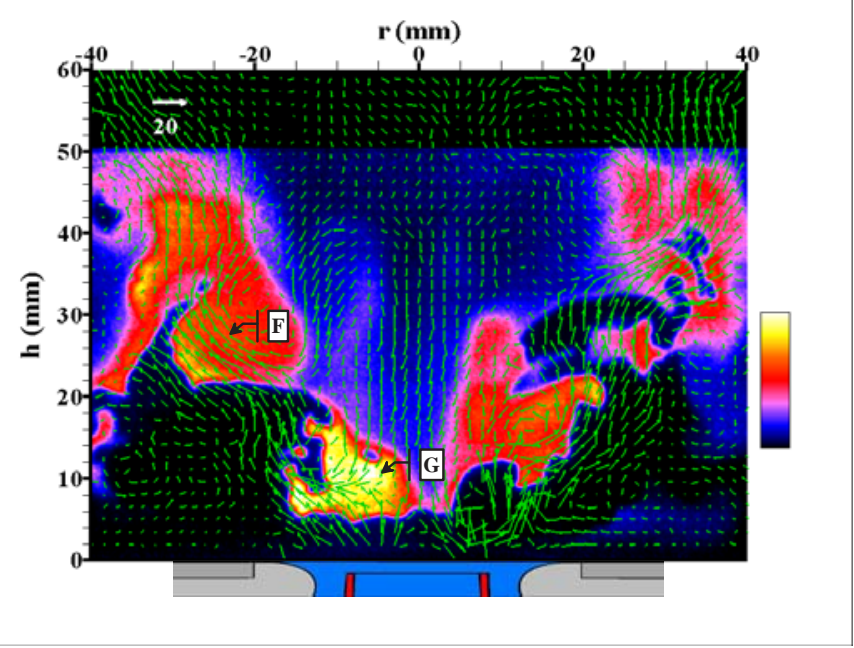

Fig. 10 Simultaneous OH-PLIF and velocity distributions.

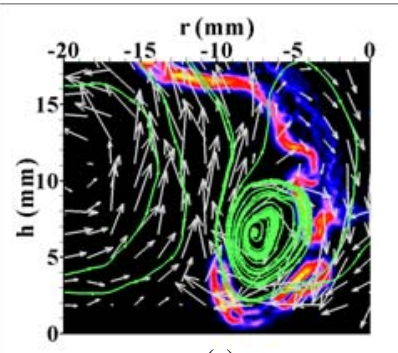

(a)

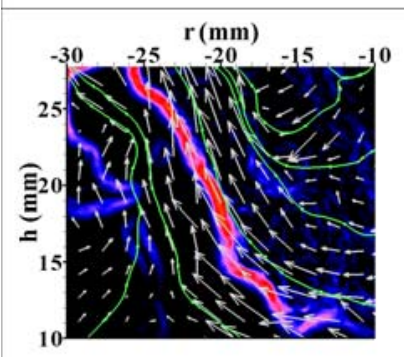

(c)

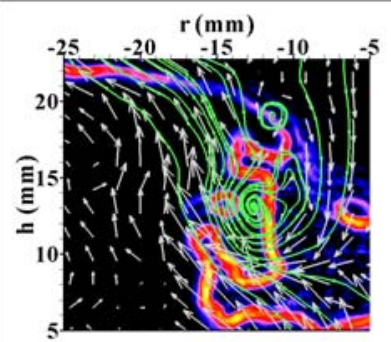

(b)

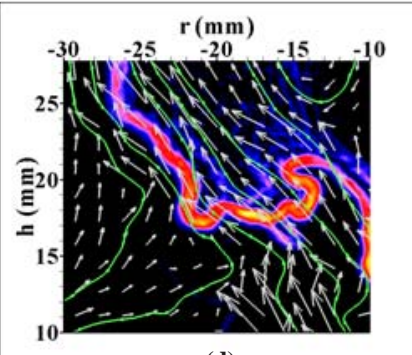

(d)
Fig. 11 Examples showing the influence of flow dynamics on reaction zone orientation.

\section{Summary and Conclusions}

Simultaneous OH-PLIF and PIV measurements have been performed in a gas turbine model combustor with good optical access, operating under atmospheric conditions. A confined swirling $\mathrm{CH}_{4}$ /air flame with an overall equivalence ratio of 0.75 and a thermal power of $10.3 \mathrm{~kW}$ was investigated. The time averaged streamline images showed flowfields typical of enclosed swirl burners, namely an inner recirculation zone and an outer recirculation zone. However, the instantaneous stream line images showed the existence of small vortical structures close to the shear layers. The approximate position and structure of the transient reaction zone in the combustor was identified by deducing the gradient of the $\mathrm{OH}$ -
PLIF images. This deduction process makes use of the existence of superequilibrium $\mathrm{OH}$ radicals at high concentrations in the reaction zone, creating a high $\mathrm{OH}$ concentration gradient along the reaction zone during the gradient calculation process, thereby effectively marking the position and structure of the reaction zone.

Reaction zones were predominantly formed at the outer or inner shear layers where the hot burned gas from the outer or inner recirculation zones mixes with the fresh fuel/air mixture from the burner nozzle. This intense mixing between the hot and cold gases was seen to play a vital role in the stabilisation of the flame at regions of high flow velocities. The flame front in the instantaneous OH-PLIF images are strongly wrinkled reflecting the strong local turbulence present. Though the reaction zones were continuous over some centimeters, isolated regions, presumably of ignition and extinction, were often visible in the single shot OH-PLIF images, emphasizing the existence of finite rate chemistry effects. Numerical simulations were performed using the PREMIX code with the GRI 3.0 mechanism to evaluate the dependence of superequilibrium $\mathrm{OH}$ concentrations on equivalence ratios and mixture temperature. The simulations showed that the $\mathrm{OH}$ levels depend mainly on local $\phi$ and less on the degree of mixing between fresh and burned gas. Superequilibrium $\mathrm{OH}$ concentration up to approximately 5 times the equilibrium value are likely to occur in this type of flame. From the simultaneous measurements it was seen that the small scale vortical structures close to the shear layer sometimes influences the reaction zone structure by rolling up the reaction zone. However, this process seem to depend greatly on the strength of the vortices as weak vortices did not have any influence the reaction zone structure. Though the orientation of the reaction zone was parallel to the flowfield in most of the 2D images, cases where the orientation of the reaction zone being perpendicular to the flowfield were also visible in some regions of the flow. Considering the large variety of thermochemical states present in this flame, as known from previous Raman measurements, and the highly corrugated turbulent flowfield structure, it becomes obvious that this type of flames are governed by a rather complex interplay between turbulence and chemical kinetics.

Acknowledgements The work has been supported under the DLR research project "Mehrskalen Verbrennungs Simulation" (MVS). The authors would also like to thank Dr. Marina Braun-Unkhoff for performing the calculations with PREMIX.

\section{References}

1. W. Meier, P. Weigand, X.R.Duan, R. Giezendanner-Thoben, Combustion and Flame 150 (2007) 2-26.

2. K.J. Syed, E,Buchanan, Proc. ASME Turbo Expo, GT-200568070 (2005).

3. W. Meier, X.R. Duan, P. Weigand, Combustion and Flame 144 (2006) 225-236.

4. J.J. Keller, AIAA Journal 33 (1995) 2280-2287. 
5. A.H. Lefebvre, Gas Turbine Combustion (Taylor \& Francis, Philadelphia 1999).

6. Th. Sattelmayer, Journal of Engineering for Gas Turbines and Power 125 (2003) 11-19.

7. T. Lieuwen, Turbomachinery International 44 (2003) 16-19.

8. N. Syred, Progress in Energy Combustion Science 32 (2006) 93161.

9. K. Kohse-Höinghaus, J. Jeffries (Eds.), Applied Combustion Diagnostics (Taylor \& Francis, New York 2002).

10. A.C. Eckbreth, Laser Diagnostic for Combustion Temperature and Species (Gordon and Breach, Netherlands 1996).

11. J.J. Frank, P.A.M. Kalt, R.W. Bilger, Combustion and Flame 116 (1999) 220-232.

12. B. Böhm, D. Geyer, A. Dreizler, K.K. Venkatesan, N.M. Laurendeau, M.W. Renfro, Proceedings of the Combustion Institute 31 (2007) 709-717.

13. T.R. Meyer, G.J. Fiechtner, S.P. Gogineni, J.C. Rolon, C.D. Carter, J.R. Gord, Experiments in Fluids 36 (2004) 259-267.

14. C.D. Carter, J.M. Donbar, J.F. Driscoll, Applied Physics B 66 (1998) 129-132.

15. J.M. Donbar, J.F. Driscoll, C.D. Carter, Combustion and Flame 125 (2001) 1239-1257.

16. J.E. Rehm, N.T. Clemens, Proceedings of the Combustion Institute 27 (1998) 1113-1120.

17. D. Han, M.G. Mungal, Combustion and Flame 132 (2003) 565590.

18. K.A. Watson, K.M. Lyons, J.M. Donbar, C.D. Carter, Combustion and Flame 117 (1999) 257-271.

19. J. Hult, U. Meier, W. Meier, A. Harvey, C.F. Kaminski, Proceedings of the Combustion Institute 30 (2005) 701-709.

20. P. Petersson, J. Olofsson, C. Brackman, H. Seyfried, J. Zetterberg, M. Richter, M. Alden, M.A. Linne, R.K. Cheng, A. Nauert, D. Geyer, A. Dreizler, Applied Optics, 46 (2007) 3928-3936.

21. M. Tanahashi, S. Murakami, G.-M. Choi, Y.Fukuchi, T. Miyauchi, Proceedings of the Combustion Institute 30 (2005) 1665-1672.

22. R. Giezendanner-Thoben, O. Keck, P. Weigand, W. Meier, U. Meier, W. Stricker, M. Aigner, Combustion Science and Technology 175 (2003) 721-741.

23. X.R. Duan, P. Weigand, W. Meier, O. Keck, B. Lehmann, W. Stricker, M. Aigner, Progress in Computational Fluid Dynamics 4 (2004) 175-184.

24. W. Meier, X.R. Duan, P. Weigand, Proceedings of the Combustion Institute 30 (2005) 835-842.

25. X.R. Duan, W. Meier, P. Weigand, B. Lehmann, Applied Physics B 80 (2005) 389-396.

26. R. Giezendanner-Thoben, P. Weigand, X.R. Duan, W. Meier, U. Meier, M. Aigner, B. Lehmann, Journal of Engineering for Gas Turbines and Power, 127 (2005) 492-496.

27. P. Weigand, W. Meier, X.R. Duan, R. Giezendanner-Thoben, U. Meier, Flow, Turbulence and Combustion, 75 (2005) 275-292.

28. R. Giezendanner-Thoben, U. Meier, W. Meier, M. Aigner, Flow, Turbulence and Combustion, 75 (2005) 317-333.

29. R. Giezendanner-Thoben, U. Meier, W. Meier, J. Heinze, M. Aigner, Applied Optics 44 (2005) 6565-6577.

30. P. Weigand, W. Meier, X.R.Duan, W. Stricker, M. Aigner, Combustion and Flame 144 (2006) 205-224.

31. M. Cao, H. Eichhoff, F. Joos, B. Simon, ASME Propulsion and Energetics, 70th Symposium, AGARD Conference Proceedings 422 (1987) 8.1.

32. J. Tobai, T. Dreier, J.W. Daily, Journal of Chemical Physics, 116 (2002) 4030-4038.
33. M.C. Drake, R.W. Pitz, M. Lapp, C.P. Fenimore, R.P. Lucht, D.W. Sweeney, N.M. Laurendeau, Proceedings of the Combustion Institute 20 (1984) 327-335.

34. R.S. Barlow, R.W. Dibble, J.-Y. Chen, R.P. Lucht, Combustion and Flame 82 (1990) 235-251.

35. B. Jähne, Digital Image Processing (Springer, Berlin 2005).

36. R. Giezendanner-Thoben, Ph.D thesis, University of Stuttgart (2006).

37. C.J. Mueller, J.F. Driscoll, D.L. Reuss, M.C. Drake, M.E. Rosalik, Combustion and Flame 112 (1998) 342-358. 